PROCEEDINGS OF THE

AMERICAN MATHEMATICAL SOCIETY

Volume 134, Number 9, September 2006, Pages 2599-2605

S 0002-9939(06)08271-2

Article electronically published on February 17, 2006

\title{
COMPOSITION OPERATORS WITH MAXIMAL NORM ON WEIGHTED BERGMAN SPACES
}

\author{
BRENT J. CARSWELL AND CHRISTOPHER HAMMOND
}

(Communicated by Joseph A. Ball)

\begin{abstract}
We prove that any composition operator with maximal norm on one of the weighted Bergman spaces $A_{\alpha}^{2}$ (in particular, on the space $A^{2}=A_{0}^{2}$ ) is induced by a disk automorphism or a map that fixes the origin. This result demonstrates a major difference between the weighted Bergman spaces and the Hardy space $H^{2}$, where every inner function induces a composition operator with maximal norm.
\end{abstract}

\section{INTRODUCTION}

Let $\mathbb{D}$ denote the open unit disk in the complex plane, with $d A$ signifying normalized area measure on $\mathbb{D}$. Recall that the Hardy space $H^{2}$ is the set of all analytic functions $f$ on $\mathbb{D}$ such that

$$
\|f\|_{H^{2}}^{2}:=\sup _{0<r<1} \frac{1}{2 \pi} \int_{0}^{2 \pi}\left|f\left(r e^{i \theta}\right)\right|^{2} d \theta<\infty .
$$

For $\alpha>-1$, the weighted Bergman space $A_{\alpha}^{2}$ consists of all analytic $f$ on $\mathbb{D}$ for which

$$
\begin{aligned}
\|f\|_{\alpha}^{2}: & =\int_{\mathbb{D}}|f(z)|^{2}(\alpha+1)\left(1-|z|^{2}\right)^{\alpha} d A(z) \\
& =\frac{\alpha+1}{\pi} \int_{0}^{1} r\left(1-r^{2}\right)^{\alpha}\left(\int_{0}^{2 \pi}\left|f\left(r e^{i \theta}\right)\right|^{2} d \theta\right) d r
\end{aligned}
$$

is finite. The space $A_{0}^{2}$, usually denoted $A^{2}$, is called the (unweighted) Bergman space. The Hardy space $H^{2}$ and the weighted Bergman spaces $A_{\alpha}^{2}$ are all Hilbert spaces, each under the obvious inner product. The properties of these spaces are discussed further in [5, 6], and 9].

Let $\varphi$ be an analytic self-map of $\mathbb{D}$. If $\mathcal{H}$ is a Hilbert space of analytic functions on $\mathbb{D}$, we define the composition operator $C_{\varphi}$ on $\mathcal{H}$ by the rule $C_{\varphi}(f)=f \circ \varphi$. It is well known that any $\varphi$ induces a bounded composition operator on $H^{2}$, with

$$
\frac{1}{1-|\varphi(0)|^{2}} \leq\left\|C_{\varphi}: H^{2} \rightarrow H^{2}\right\|^{2} \leq \frac{1+|\varphi(0)|}{1-|\varphi(0)|} .
$$

Received by the editors February 2, 2005 and, in revised form, March 21, 2005

2000 Mathematics Subject Classification. Primary 47B33.

Key words and phrases. Composition operator, norm, essential norm.

(C)2006 American Mathematical Society Reverts to public domain 28 years from publication 
(See [4, Corollary 3.7].) Likewise, every composition operator is bounded on each of the weighted Bergman spaces $A_{\alpha}^{2}$, with

$$
\left(\frac{1}{1-|\varphi(0)|^{2}}\right)^{\alpha+2} \leq\left\|C_{\varphi}: A_{\alpha}^{2} \rightarrow A_{\alpha}^{2}\right\|^{2} \leq\left(\frac{1+|\varphi(0)|}{1-|\varphi(0)|}\right)^{\alpha+2} .
$$

(The proof of (2) is similar to that of (11); see, for example, 16, Lemma 2.3].) When $\varphi(0)=0$, it is easy to see that the norm of the corresponding composition operator, acting on either the Hardy space or one of weighted Bergman spaces, is equal to 1. When $\varphi(0) \neq 0$, on the other hand, it is quite difficult to determine the norm of $C_{\varphi}$ exactly. (See [1], 2], 7], 8, 10], and [13] for explicit norm calculations; related results appear in [3, [15], 16], [17, 19, [22], and [23.)

One problem that has attracted significant attention is the question of which composition operators have maximal norm on a particular Hilbert space. In other words, for the Hardy space $H^{2}$, one would like to identify the maps $\varphi: \mathbb{D} \rightarrow \mathbb{D}$ for which

$$
\left\|C_{\varphi}: H^{2} \rightarrow H^{2}\right\|^{2}=\frac{1+|\varphi(0)|}{1-|\varphi(0)|} .
$$

Similarly, for a weighted Bergman space $A_{\alpha}^{2}$, one would like to determine when

$$
\left\|C_{\varphi}: A_{\alpha}^{2} \rightarrow A_{\alpha}^{2}\right\|^{2}=\left(\frac{1+|\varphi(0)|}{1-|\varphi(0)|}\right)^{\alpha+2} .
$$

This question has already been answered in the context of the Hardy space. In one of the earliest papers on composition operators, Nordgren [13] showed that $C_{\varphi}: H^{2} \rightarrow H^{2}$ has maximal norm whenever $\varphi$ is an inner function; that is, when-

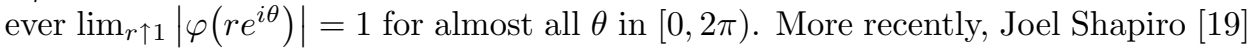
demonstrated that, when $\varphi(0) \neq 0$, these are the only composition operators with maximal norm on $H^{2}$. (See 12 for an alternate approach to this result.) Until now, this problem has remained open in the setting of the weighted Bergman spaces, even for the space $A^{2}=A_{0}^{2}$. It is already known that any disk automorphism induces a composition operator with maximal norm on $A_{\alpha}^{2}$ (see, for example, the proof of [16. Lemma 2.3]). The purpose of this paper is to prove that, when $\varphi(0) \neq 0$, there are no other composition operators that have maximal norm on $A_{\alpha}^{2}$. This result is the substance of Theorem 3.3 .

We remark that the complementary question - which composition operators have minimal norm - can be answered without much difficulty. In fact, when $\varphi(0) \neq 0$, the only composition operators with minimal norm, either on the Hardy space or one of the weighted Bergman spaces, are those with constant symbol (see [7, Lemma 4.1], 8, Lemma 2.1], [15, Theorem 4], and [22, Theorem 3]).

\section{ESSENTIAL NORMS}

Let $\mathcal{H}$ be a Hilbert space. Recall that the essential norm $\|T\|_{e}$ of an operator $T: \mathcal{H} \rightarrow \mathcal{H}$ is defined as follows:

$$
\|T\|_{e}=\inf _{K}\|T-K\|
$$

the infimum being taken over the set of all compact operators $K: \mathcal{H} \rightarrow \mathcal{H}$. In other words, the essential norm represents the distance between $T$ and the set of compact operators. It is often helpful to consider the relationship between the norm of an operator and its essential norm. Since the trivial operator is compact, we see that 
$\|T\|_{e} \leq\|T\|$ for any $T: \mathcal{H} \rightarrow \mathcal{H}$. Furthermore, if $\|T\|_{e}<\|T\|$, we know that the operator $T$ is norm-attaining (see [7, Proposition 2.2]); that is, there is some unit vector $h$ in $\mathcal{H}$ such that $\|T(h)\|=\|T\|$. We will make use of this fact in the proof of Proposition 3.1

Now consider a composition operator $C_{\varphi}$, acting on either $H^{2}$ or $A_{\alpha}^{2}$. It makes sense to say that $C_{\varphi}$ has maximal essential norm whenever line (3) or line (44), as appropriate, holds with the norm of $C_{\varphi}$ replaced by the essential norm. As it turns out, our investigation of composition operators with maximal norm will also allow us to characterize the composition operators that have maximal essential norm on $A_{\alpha}^{2}$. We note that this condition is not equivalent to saying that $\left\|C_{\varphi}\right\|_{e}=\left\|C_{\varphi}\right\|$, a property often called extremal noncompactness. When $\varphi(z)=(1 / 2) z+1 / 2$, for example, one can show that $C_{\varphi}: A_{\alpha}^{2} \rightarrow A_{\alpha}^{2}$ is extremally noncompact, but that it does not have maximal essential norm.

As it happens, more information is known about the essential norm of a composition operator than about the norm itself. In particular, we have explicit formulas for the essential norm of $C_{\varphi}$ acting on a variety of spaces. The prototypical result, a formula for the essential norm of a composition operator on the Hardy space, was established by Joel Shapiro [18. His technique was later adapted to the weighted Bergman spaces by Poggi-Corradini 14, who obtained a formula for $\left\|C_{\varphi}: A_{\alpha}^{2} \rightarrow A_{\alpha}^{2}\right\|_{e}$ when $\alpha=0$ or $\alpha=1$. Poggi-Corradini's proof relies on the use of contractive zero-divisors for the spaces $A^{2}$ and $A_{1}^{2}$. Results due to Shimorin 20, 21, pertaining to contractive divisors, show that Poggi-Corradini's formula is actually valid whenever $-1<\alpha \leq 1$.

In the course of proving his essential norm formula, Poggi-Corradini demonstrated the following result, which holds for all $\alpha>-1$ :

$$
\left\|C_{\varphi}: A_{\alpha}^{2} \rightarrow A_{\alpha}^{2}\right\|_{e}^{2} \leq \limsup _{|a| \rightarrow 1} \frac{\sum_{z_{j} \in \varphi^{-1}(a)} W\left(\left|z_{j}\right|\right)}{W(|a|)}
$$

where

$$
W(r)=\frac{\left(1-r^{2}\right)^{\alpha+2}}{\alpha+2} .
$$

This fact is a major ingredient in the proof of [14, Claim 3.1]. Cowen and MacCluer, as part of the proof of [4. Corollary 3.21], showed that

$$
\limsup _{|a| \rightarrow 1} \frac{\sum_{z_{j} \in \varphi^{-1}(a)} H\left(\left|z_{j}\right|\right)}{H(|a|)} \leq \frac{1+|\varphi(0)|}{1-|\varphi(0)|}\left(\frac{1}{\beta(\varphi)}\right)^{\alpha+1},
$$

where $H(r)=\left|\log \left(r^{2}\right)\right|^{\alpha+2}$ and $\beta(\varphi)=\liminf _{|z| \rightarrow 1}(1-|\varphi(z)|) /(1-|z|)$. It is not difficult to see that

$$
\limsup _{|a| \rightarrow 1} \frac{\sum_{z_{j} \in \varphi^{-1}(a)} W\left(\left|z_{j}\right|\right)}{W(|a|)}=\limsup _{|a| \rightarrow 1} \frac{\sum_{z_{j} \in \varphi^{-1}(a)} H\left(\left|z_{j}\right|\right)}{H(|a|)} .
$$

Consequently

$$
\left\|C_{\varphi}: A_{\alpha}^{2} \rightarrow A_{\alpha}^{2}\right\|_{e}^{2} \leq \frac{1+|\varphi(0)|}{1-|\varphi(0)|}\left(\frac{1}{\beta(\varphi)}\right)^{\alpha+1}
$$

for any analytic $\varphi: \mathbb{D} \rightarrow \mathbb{D}$. Suppose then that $\varphi(0)=0$; in this case, Lemma 7.33 in [4] dictates that $\beta(\varphi)>1$ whenever $\varphi$ is not a rotation. In other words, we have established the following proposition. 
Proposition 2.1. Let $\varphi$ be an analytic self-map of $\mathbb{D}$ with $\varphi(0)=0$. If $\varphi$ is not a rotation, then $\left\|C_{\varphi}: A_{\alpha}^{2} \rightarrow A_{\alpha}^{2}\right\|_{e}<1$.

This observation will allow us to obtain the major results of Section 3 ,

We conclude this section by mentioning a class of subspaces that have proved particularly useful to the study of essential norms. For any natural number $m$, let $z^{m} A_{\alpha}^{2}$ denote the subspace consisting of all functions in $A_{\alpha}^{2}$ with a zero of order at least $m$ at the origin (with $z A_{\alpha}^{2}=z^{1} A_{\alpha}^{2}$ ). When $\varphi(0)=0$, each of these subspaces is invariant under the operator $C_{\varphi}$. In this situation, we write $\left\|C_{\varphi}\right\|_{(m)}$ to denote the norm of $C_{\varphi}: z^{m} A_{\alpha}^{2} \rightarrow z^{m} A_{\alpha}^{2}$. It is well known that the values $\left\|C_{\varphi}\right\|_{(m)}$ form a nonincreasing sequence, with $\left\|C_{\varphi}\right\|_{e}=\lim _{m \rightarrow \infty}\left\|C_{\varphi}\right\|_{(m)}$ (see [18, Proposition 5.1]). One consequence of this fact is that the essential norm of $C_{\varphi}: z^{m} A_{\alpha}^{2} \rightarrow z^{m} A_{\alpha}^{2}$, for any $m$, is equal to the essential norm of $C_{\varphi}: A_{\alpha}^{2} \rightarrow A_{\alpha}^{2}$. As we shall see, much of our investigation will take place in the setting where $\varphi(0)=0$. Our results will depend on the relationship between the quantities $\left\|C_{\varphi}\right\|,\left\|C_{\varphi}\right\|_{(1)}$, and $\left\|C_{\varphi}\right\|_{e}$.

\section{THE MAIN THEOREM}

We begin with the following proposition, which can be seen as an analogue of Theorem 5.1 in [19].

Proposition 3.1. Let $\varphi$ be an analytic self-map of $\mathbb{D}$ with $\varphi(0)=0$. If $\varphi$ is not a rotation, then $\left\|C_{\varphi}\right\|_{(1)}<1$.

Proof. Suppose that $\varphi$ is not a rotation. Proposition 2.1 dictates that $\left\|C_{\varphi}\right\|_{e}<1$. If $\left\|C_{\varphi}\right\|_{(1)}=\left\|C_{\varphi}\right\|_{e}$, then our claim follows automatically. Suppose then that $\left\|C_{\varphi}\right\|_{(1)}>\left\|C_{\varphi}\right\|_{e}$. In this case, the operator $C_{\varphi}: z A_{\alpha}^{2} \rightarrow z A_{\alpha}^{2}$ is norm-attaining; that is, there is a unit vector $f$ in $z A_{\alpha}^{2}$ with $\left\|C_{\varphi}\right\|_{(1)}=\left\|C_{\varphi}(f)\right\|_{\alpha}$. Examining the proof of the Littlewood Subordination Theorem (see [4, p. 30]), we observe that

$$
\int_{0}^{2 \pi}\left|f\left(\varphi\left(r e^{i \theta}\right)\right)\right|^{2} d \theta<\int_{0}^{2 \pi}\left|f\left(r e^{i \theta}\right)\right|^{2} d \theta
$$

for any $0<r<1$, unless either $f$ is constant or $\varphi$ is a rotation. (See also [4, Exercise 2.2.1].) We have assumed that $\varphi$ is not a rotation; since $f$ belongs to $z A_{\alpha}^{2}$, it cannot be constant. Therefore $\left\|C_{\varphi}\right\|_{(1)}=\left\|C_{\varphi}(f)\right\|_{\alpha}<\|f\|_{\alpha}=1$, as we had hoped to show.

One consequence of this argument is that $C_{\varphi}: A_{\alpha}^{2} \rightarrow A_{\alpha}^{2}$ is an isometry if and only if $\varphi$ is a rotation; this fact differs somewhat from the analogous results that are known for other Hilbert spaces. For example, $C_{\varphi}$ acts isometrically on $H^{2}$ if and only if $\varphi$ is an inner function that fixes the origin (see [13] and 19]). There is yet a different characterization (recently obtained by Martín and Vukotić [11]) for the isometric composition operators on the Dirichlet space.

For any point $w$ in $\mathbb{D}$, consider the map

$$
\psi_{w}(z)=\frac{w-z}{1-\bar{w} z},
$$

the involutive automorphism that interchanges the points 0 and $w$. If $\varphi$ is a selfmap of $\mathbb{D}$, we define the Frostman transform $\varphi_{w}$ to be the composition $\psi_{w} \circ \varphi$. If $w=\varphi(0)$, then the map $\varphi_{w}$ fixes the origin. Our next result can be obtained by 
making the obvious modifications to the proof of [19, Theorem 5.2], which provides an analogous inequality for composition operators acting on $H^{2}$.

Proposition 3.2. Let $\varphi$ be an analytic self-map of $\mathbb{D}$ with $\varphi(0)=w$. Then

$$
\left\|C_{\varphi}: A_{\alpha}^{2} \rightarrow A_{\alpha}^{2}\right\|^{2} \leq\left(\nu+\frac{1-\nu}{(1+|w|)^{2 \alpha+4}}\right)\left(\frac{1+|w|}{1-|w|}\right)^{\alpha+2},
$$

where $\nu=\left\|C_{\varphi_{w}}\right\|_{(1)}^{2}$.

Proof. For the sake of completeness, we reproduce Shapiro's argument. Let $f$ be a unit vector in $A_{\alpha}^{2}$. Since $\varphi=\psi_{w} \circ \varphi_{w}$, we see that

$$
C_{\varphi}(f)=C_{\varphi_{w}}\left(f \circ \psi_{w}\right)=C_{\varphi_{w}}(g)+f(w),
$$

where $g=f \circ \psi_{w}-f(w)$. Note that $C_{\varphi_{w}}(g)$ vanishes at the origin, and hence is orthogonal to the constant $f(w)$; therefore

$$
\left\|C_{\varphi}(f)\right\|_{\alpha}^{2}=\left\|C_{\varphi_{w}}(g)\right\|_{\alpha}^{2}+|f(w)|^{2} .
$$

Since $g$ belongs to $z A_{\alpha}^{2}$, we see that

$$
\left\|C_{\varphi_{w}}(g)\right\|_{\alpha}^{2} \leq\left\|C_{\varphi_{w}}\right\|_{(1)}^{2}\|g\|_{\alpha}^{2}=\nu\|g\|_{\alpha}^{2} .
$$

Furthermore,

$$
\begin{aligned}
\|g\|_{\alpha}^{2} & =\left\|f \circ \psi_{w}-f(w)\right\|_{\alpha}^{2}=\left\|f \circ \psi_{w}\right\|_{\alpha}^{2}-2 \operatorname{Re} \overline{f(w)}\left\langle f \circ \psi_{w}, 1\right\rangle+|f(w)|^{2} \\
& =\left\|f \circ \psi_{w}\right\|_{\alpha}^{2}-2 \operatorname{Re} \overline{f(w)} f(w)+|f(w)|^{2}=\left\|C_{\psi_{w}}(f)\right\|_{\alpha}^{2}-|f(w)|^{2} .
\end{aligned}
$$

Since $\psi_{w}$ is an automorphism of $\mathbb{D}$, we know that

$$
\left\|C_{\psi_{w}}\right\|^{2}=\left(\frac{1+|w|}{1-|w|}\right)^{\alpha+2} .
$$

A well-known estimate (see [9, Lemma 3.2]) dictates that $|f(w)|^{2} \leq 1 /\left(1-|w|^{2}\right)^{\alpha+2}$. Consequently

$$
\begin{aligned}
\left\|C_{\varphi}(f)\right\|_{\alpha}^{2} & \leq \nu\|g\|_{\alpha}^{2}+|f(w)|^{2}=\nu\left(\left\|C_{\psi_{w}}(f)\right\|_{\alpha}^{2}-|f(w)|^{2}\right)+|f(w)|^{2} \\
& =\nu\left\|C_{\psi_{w}}(f)\right\|_{\alpha}^{2}+(1-\nu)|f(w)|^{2} \\
& \leq \nu\left(\frac{1+|w|}{1-|w|}\right)^{\alpha+2}+\frac{1-\nu}{\left(1-|w|^{2}\right)^{\alpha+2}} \\
& =\left(\nu+\frac{1-\nu}{(1+|w|)^{2 \alpha+4}}\right)\left(\frac{1+|w|}{1-|w|}\right)^{\alpha+2}
\end{aligned}
$$

as we had hoped to show.

In particular, if $w=\varphi(0) \neq 0$ and $\left\|C_{\varphi_{w}}\right\|_{(1)}<1$, we see that $\left\|C_{\varphi}\right\|$ is not maximal. In other words, we have established our main result.

Theorem 3.3. Consider the weighted Bergman space $A_{\alpha}^{2}$, with $\alpha>-1$. Let $\varphi$ be an analytic self-map of $\mathbb{D}$. If $\varphi(0) \neq 0$, the following conditions are equivalent:

(1) The operator $C_{\varphi}: A_{\alpha}^{2} \rightarrow A_{\alpha}^{2}$ has maximal norm.

(2) The operator $C_{\varphi}: A_{\alpha}^{2} \rightarrow A_{\alpha}^{2}$ has maximal essential norm.

(3) The map $\varphi: \mathbb{D} \rightarrow \mathbb{D}$ is an automorphism. 
Proof. (3) $\Rightarrow(2)$ Suppose that $\varphi$ is an automorphism; it follows from 4, Proposition 3.13] that

$$
\left\|C_{\varphi}\right\|_{e}^{2} \geq \limsup _{|a| \rightarrow 1}\left(\frac{1-|a|^{2}}{1-|\varphi(a)|^{2}}\right)^{\alpha+2}=\left(\frac{1+|\varphi(0)|}{1-|\varphi(0)|}\right)^{\alpha+2} .
$$

In other words, the operator $C_{\varphi}$ has maximal essential norm.

(2) $\Rightarrow(1)$ Since $\left\|C_{\varphi}\right\|_{e} \leq\left\|C_{\varphi}\right\|$, any composition operator with maximal essential norm also has maximal norm.

$(1) \Rightarrow(3)$ Let $w=\varphi(0)$. If $\varphi$ is not an automorphism, then the map $\varphi_{w}=\psi_{w} \circ \varphi$ is not a rotation. Thus Proposition 3.1 shows that $\left\|C_{\varphi_{w}}\right\|_{(1)}<1$, so it follows from Proposition 3.2 that $C_{\varphi}: A_{\alpha}^{2} \rightarrow A_{\alpha}^{2}$ does not have maximal norm.

\section{NORMS ON DIFFERENT SPACES}

It is an interesting exercise to compare the norm of a composition operator acting on the unweighted Bergman space $A^{2}$ to its norm on the Hardy space $H^{2}$. Considering lines (1) and (2), one might be tempted to speculate that

$$
\left\|C_{\varphi}: A^{2} \rightarrow A^{2}\right\|=\left\|C_{\varphi}: H^{2} \rightarrow H^{2}\right\|^{2} .
$$

In fact, this equality does hold in most of the cases where we know how to calculate both quantities explicitly. Nonetheless, it is possible to find examples of $\varphi: \mathbb{D} \rightarrow \mathbb{D}$ for which equation (5) is false. In particular, let

$$
\varphi(z)=\frac{(r+s) z+(1-s)}{r(1-s) z+(1+s r)}
$$

for $0<s<1$ and $-1 / 7 \leq r<0$. In this case, Richman [16, Theorem 4.1] demonstrated that the operator $C_{\varphi}: A^{2} \rightarrow A^{2}$ has subnormal adjoint; hence its norm is equal to its spectral radius, which is $1 / \varphi^{\prime}(1)=1 / s$. On the other hand, Bourdon et al. [1, Theorem 3.7] showed that the norm of $C_{\varphi}: H^{2} \rightarrow H^{2}$ is strictly greater than $\sqrt{1 / s}$. Likewise, let $\varphi$ be a nonunivalent inner function with $\varphi(0) \neq$ 0 . Then, as we have already stated, the corresponding composition operator has maximal norm on $H^{2}$. Theorem 3.3 , which we have just proved, shows that $C_{\varphi}$ does not have the same property on $A^{2}$.

Both examples from the preceding paragraph demonstrate that it is possible for $\left\|C_{\varphi}: A^{2} \rightarrow A^{2}\right\|$ to be strictly less than $\left\|C_{\varphi}: H^{2} \rightarrow H^{2}\right\|^{2}$. In light of this observation, it would be interesting to determine whether the inequality

$$
\left\|C_{\varphi}: A^{2} \rightarrow A^{2}\right\| \leq\left\|C_{\varphi}: H^{2} \rightarrow H^{2}\right\|^{2}
$$

holds for every analytic $\varphi: \mathbb{D} \rightarrow \mathbb{D}$. This is an open question that we commend to the reader's attention. We remark that the analogous inequality does indeed hold for essential norms, a fact that can be deduced simply by comparing the relevant formulas (14, Theorem 1.1] and [18, Theorem 2.3]).

\section{REFERENCES}

[1] P. S. Bourdon, E. E. Fry, C. Hammond, and C. H. Spofford, Norms of linear-fractional composition operators, Trans. Amer. Math. Soc. 356 (2004), 2459-2480. MR2048525 (2004m:47045)

[2] C. C. Cowen, Linear fractional composition operators on $H^{2}$, Integral Equations Operator Theory 11 (1988), 151-160. MR0928479 (89b:47044) 
[3] C. C. Cowen and T. L. Kriete, Subnormality and composition operators on $H^{2}$, J. Funct. Anal. 81 (1988), 298-319. MR0971882 (90c:47055)

[4] C. C. Cowen and B. D. MacCluer, Composition Operators on Spaces of Analytic Functions, CRC Press, Boca Raton, 1995. MR,1397026 (97i:47056)

[5] P. L. Duren, Theory of $H^{p}$ Spaces, Academic Press, New York, 1970. MR0268655(42:3552)

[6] P. Duren and A. Schuster, Bergman Spaces, American Mathematical Society, Providence, 2004. MR2033762 (2005c:30053)

[7] C. Hammond, On the norm of a composition operator with linear fractional symbol, Acta Sci. Math. (Szeged) 69 (2003), 813-829. MR2034210 (2004m:47049)

[8] C. Hammond, On the norm of a composition operator, Ph.D. thesis, University of Virginia, 2003.

[9] H. Hedenmalm, B. Korenblum, and K. Zhu, Theory of Bergman Spaces, Springer-Verlag, New York, 2000. MR1758653(2001c:46043)

[10] P. R. Hurst, Relating composition operators on different weighted Hardy spaces, Arch. Math. (Basel) 68 (1997), 503-513. MR.1444662 (98c:47040)

[11] M. J. Martín and D. Vukotić, Isometries of the Dirichlet space among the composition operators, Proc. Amer. Math. Soc., to appear.

[12] V. Matache, A short proof of a characterization of inner functions in terms of the composition operators they induce, Rocky Mountain J. Math. 35 (2005), 1723-1726.

[13] E. A. Nordgren, Composition operators, Canad. J. Math. 20 (1968), 442-449. MR0223914 (36:6961)

[14] P. Poggi-Corradini, The essential norm of composition operators revisited, Studies on Composition Operators (Laramie, 1996), 167-173, Contemp. Math., 213, Amer. Math. Soc., Providence, 1998. MR1601104 (98m:47046)

[15] D. B. Pokorny and J. E. Shapiro, Continuity of the norm of a composition operator, Integral Equations Operator Theory 45 (2003), 351-358. MR1965901 (2004b:47047)

[16] A. E. Richman, Subnormality and composition operators on the Bergman space, Integral Equations Operator Theory 45 (2003), 105-124. MR.1952344(2004c:47050)

[17] A. E. Richman, Composition operators with complex symbol having subnormal adjoint, Houston J. Math. 29 (2003), 371-384. MR1987582(2004i:47049)

[18] J. H. Shapiro, The essential norm of a composition operator, Annals Math. 125 (1987), 375-404. MR.0881273 (88c:47058)

[19] J. H. Shapiro, What do composition operators know about inner functions?, Monatsh. Math. 130 (2000), 57-70. MR:1762064 (2001a:47029)

[20] S. M. Shimorin, Factorization of analytic functions in weighted Bergman spaces (Russian), Algebra i Analiz 5 (1993), 155-177; translation in St. Petersburg Math. J. 5 (1994), 10051022. MR.1263318 (95j:30032)

[21] S. M. Shimorin, On a family of conformally invariant operators (Russian), Algebra i Analiz 7 (1995), 133-158; translation in St. Petersburg Math. J. 7 (1996), 287-306. MR 1347516 (96i:47010)

[22] D. Vukotić, On norms of composition operators acting on Bergman spaces, J. Math. Anal. Appl. 291 (2004), 189-202. MR2034066 (2004m:30058)

[23] D. Vukotić, Corrigendum to "On norms of composition operators acting on Bergman spaces" [J. Math. Anal. Appl. 291 (2004), 189-202. MR2034066 (2004m:30058)], J. Math. Anal. Appl. 311 (2005), 377-380. MR2165484

Department of Mathematics, Allegheny College, Meadville, Pennsylvania 16335

E-mail address: brent.carswell@allegheny.edu

Department of Mathematics and Computer Science, Connecticut College, New LonDON, Connecticut 06320

E-mail address: cnham@conncoll.edu 